\title{
QoS Guarantee Method for Real Time Service with Packet Priority in Home Gateway
}

\author{
Kyeong Chae Jeong*, Ju No Han*, Seong Gon Choi*† \\ ${ }^{*}$ College of Electronic and Computer Engineering Chungbuk National University \\ Cheongju, Republic of Korea \\ Email: $\{j k c$, juno8003, sgchoi $\} @$ cbnu.ac.kr
}

\begin{abstract}
In this paper, we propose a Quality of Service (QoS) method for a real time service by using priority-based packet classification method in Home Gateway (HG). A real time service is sensitive to QoS factors such as delay and jitter. On the contrary, A non-real time service is tolerant to above mentioned QoS factors. To improve QoS for the real time service, UDP is granted a high priority compared with TCP in Data Buffer Part (DBP) of HG. We analyze the expected waiting time of prioritybased packet by using queueing analysis. The result shows that a significant QoS benefit due to the reduced waiting time for the real time service.
\end{abstract}

Keywords-QoS, real time service, priority-based, packet classification, Home Gateway, waiting time

\section{INTRODUCTION}

The rapid increase in Internet data services over the past decade has led to increased traffics which consist of a real time service and a non-real time service. Due to increasing the real and non-real time service, current Home Gateway (HG) is limited in its ability to guarantee Quality of Service (QoS). HG which transfers incoming packets from Core Network $(\mathrm{CN})$ to home devices is an essential part of a local network. [1] When the packet arrives at HG, current HG is always on-line and transfer packets to home devices without considering the type of packet. Such simple processing and a structure are cause for QoS degradation.

Related to packet classification, numerous studies have been conducted to solve the QoS problem [2]-[4]. However, to the best of our knowledge, previous works have focused only on QoS enhancement, and thus insufficient attention has been focused on energy efficiency at HG. The author in [5] proposed Traffic Aware Sleep Algorithm (TASA) for energy-efficient always-on home networks. This algorithm is for HG to sleep, listen or wakeup according to network traffic adaptively. This method keeps always-on service, and minimizes the power consumption for network services. Although previous work has been conducted to analyze the energy consumption in HG, no research effort has been expended in terms of QoS of the real time service.

Thus, in this paper, we propose the QoS guarantee method for the real time service by using the packet priority at HG. Particularly, the real time service is more sensitive to QoS factors such as delay and jitter than the non-real time service. Therefore, it is necessary to classify the type of packet to reduce the waiting time for the real time service. We can improve QoS by reducing the waiting time for the real time service. We analyze the expected waiting time of priority-based packet by using queueing analysis. The result shows that a significant QoS benefit due to the reduced waiting time for the real time service.

The rest of the paper is organized as follows: Section II describes previous works related to the energy-efficient method in $\mathrm{HG}$, the signature matching method, and the queueing system. Section III presents the proposed HG structure and packet classification algorithm. The non-preemptive priority queueing analysis is presented in Section IV. Finally, the conclusion is given in Section V.

\section{RELATED WORKS}

The authors in [1] proposed Home Gateway Energy Saving Mechanism (HGESM) which reduces energy consumption in home gateway. The proposed mechanism supports energy saving by switching or keeping processor mode according to the type of service (real time or non-real time service). Namely, in sleep mode, if UDP packets are checked, HG wakes up and processes packets for minimizing its delay time. And, in active mode, if TCP packets are checked, HG decides whether keeping sleep mode or changing mode to active according to its buffer state to minimize its power consumption.

[6] proposed a traffic classification technology by using signature matching. The authors in [6] were collecting the information such as IP address, port, protocol, the string of payload, packet size and flow with this method. The network equipment monitors traffics and checks a virus and the intrusion detection using packet information and a signature. At this time, the traffic monitoring equipment needs to predefine packet field information for matching the related field of the incoming packet because the signature matching is only operated by the predefined field. As mentioned above, the signature matching technology is used for traffic monitoring or classification. At the network, a router can reduce processing time by using hardware signature matching through source IP, destination IP and protocol. That is, in order to reduce a processing time, check of useful portion of the packet is more effective than checking the whole packet [6].

The need to provide priority to certain classes of customers in a queueing system arises in many applications. Priority classes are used in many computer systems, in the computer control of digital switching exchanges, for deadlock prevention in packet-switching networks, and so forth. A simple example 


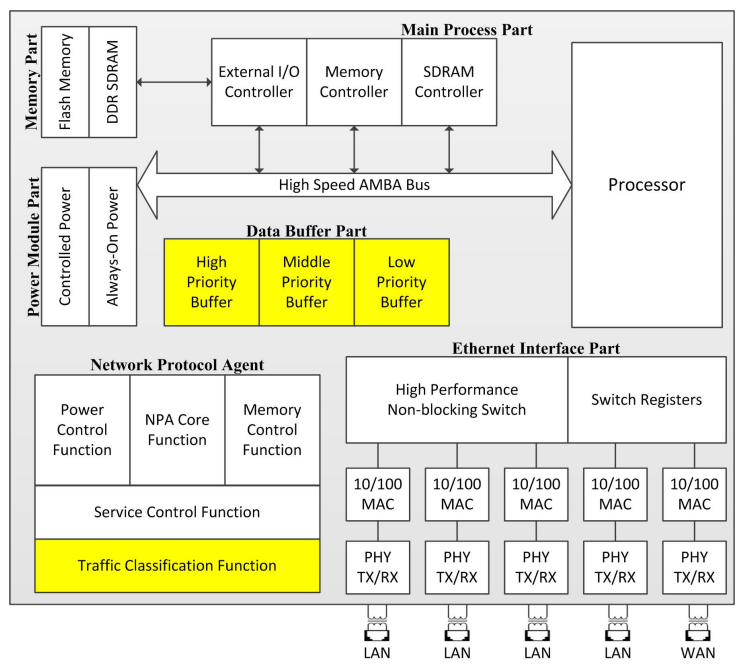

Fig. 1. Structure of Home Gateway [1]

taken from packet switching serves to provide the necessary motivation at this point. Consider a packet-switching network that transmits, in addition to the normal data packets, control packets of much shorter length that carry out the vital operations of signaling, congestion notification, fault notification, routing change information, and so on. It is vital in many cases to ensure rapid distribution of these control messages. Yet without establishing a priority, they could easily queue up behind much longer data packets, delaying their arrival at the necessary destination points [7].

In this paper, we focus on improving QoS for the real time service. To solve this problem, we propose a QoS guarantee method by using a packet priority at HG. To improve QoS for the real time service, it is necessary to classify the type of packet to reduce the waiting time for the real time service.

\section{SYSTEM MOdEL}

\section{A. QoS Guarantee Method for Real Time Service}

Priority-based packet classification is as follows: Firstly, the UDP packet related to multi streaming service and VoIP service is granted a high priority and the others among the UDP packets granted a middle priority. Lastly, the type of TCP is granted a low priority.

Fig. 1 shows the proposed structure of HG. Data Buffer Part (DBP) and Traffic Classification Function (TCF) in Network Protocol Agent (NPA) are newly included in proposed HG.

And Fig. 2 shows the flowchart of the proposed method. When the packet arrives at HG, TCF checks the type of service. In case of high priority packet, it transfers promptly without buffering. Otherwise, in case of the middle and the low priority packet, they buffered in DBP until relatively high priority packets were serviced over. By using this flowchart, we expect that the waiting time for the real time service is reduced and the waiting time for non-real time services extended. That is, we can improve QoS for the real-time service.

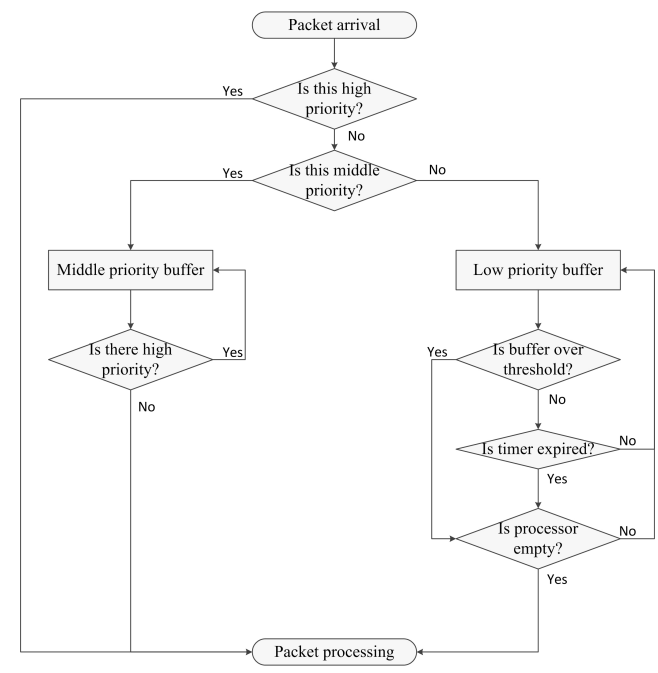

Fig. 2. Flowchart of the proposed method

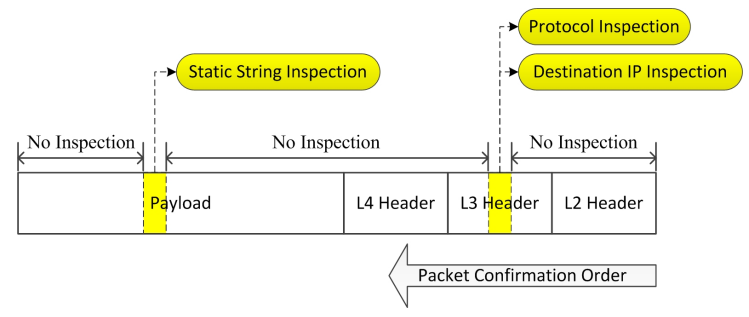

Fig. 3. Proposed traffic signature matching method

\section{B. Traffic Signature Matching Method}

Fig. 3 shows the process of confirming the packet in the signature matching method. It can reduce the waiting time for real time traffic to classify a traffic using the information such as IP address, port, protocol, the string of payload, packet size, and flow. In this work, We take a look only source and destination IP address and protocol to minimize additional delay time as shown in Fig. 3. Packets are divided into three parts the UDP packet related to multi streaming service and VoIP service, the others among the UDP packets and the type of TCP is granted a low priority.

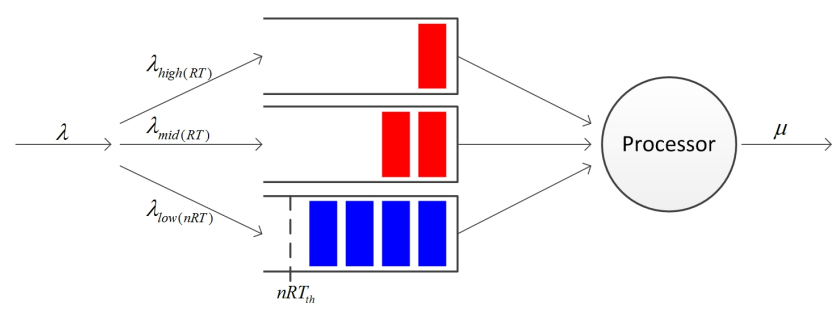

Fig. 4. Non-preemptive multi-priority queueing model 


\section{PERFORMANCE ANALYSiS}

Fig. 4 shows a non-preemptive priority queueing model for performance analysis of the proposed method. HG monitors incoming packets and classifies them according to the type of service [6]. Each waiting time in DBP can be calculated by using non-preemptive priority queueing model. Accordingly, the waiting time of the high priority packet $E\left[W_{1}\right]$ is as follows

$$
E\left[W_{1}\right]=E\left[T_{0}\right]+E\left[T_{1}\right]
$$

where $E\left[T_{0}\right]$ is processing time of traffic in server, and $E\left[T_{1}\right]$ is the waiting time of the packet which arrives previously and waits for the service. It considers processing time and the waiting time of the packet arrived previously when calculating the waiting time of the high priority packet. The waiting time of the middle priority packet $E\left[W_{2}\right]$ is as follows

$$
E\left[W_{2}\right]=E\left[T_{0}\right]+\sum_{k=1}^{2} E\left[T_{k}\right]+E\left[T_{1}^{\prime}\right]
$$

where $E\left[T_{k}\right]$ is the waiting time of the packet which arrives previously and waits for the service in each queue. $E\left[T_{1}^{\prime}\right]$ is the service time of the incoming relatively high priority packet during processing the middle priority packet. In case the waiting time of the low priority queue does not exceed the timer, the packet is served if the queue length exceeds the threshold value. The waiting time of this case is as follows

$$
E\left[W_{3}\right]=E\left[T_{0}\right]+\sum_{k=1}^{3} E\left[T_{k}\right]+\sum_{k=1}^{2} E\left[T_{k}^{\prime}\right]
$$

$E\left[T_{k}^{\prime}\right]$ is the service time of the incoming relatively high priority packet during processing the low priority packet. In this case, the queue length of the low priority queue does not exceed the threshold value, the low priority packet is delayed as smaller than timer. The delay time is according to equation (4).

$E\left[W_{3}^{\prime}\right]=E\left[T_{0}\right]+\sum_{k=1}^{3} E\left[T_{k}\right]+\sum_{k=1}^{2} E\left[T_{k}^{\prime}\right]+E\left[A_{3}\right]+\sum_{k=1}^{2} E\left[T_{k}^{\prime \prime}\right]$.

If the low priority buffer size is smaller than the threshold value, $E\left[W_{3}^{\prime}\right]$ is the waiting time of low priority packet. $E\left[A_{3}\right]$ is the delay time that the HG delays the active mode without converting its state. $E\left[T_{k}^{\prime}\right]$ is the service time of the new incoming high priority packet during the addition delay time. Average delay time $E\left[A_{3}\right]$ can be founded through the equation (5) to (9).

$$
E\left[C_{3}\right]=E\left[Q_{t h}\right]-E\left[m_{3}\right] .
$$

In equation (5), $E\left[Q_{t} h\right]$ is the selecting threshold in the low priority buffer. $E\left[m_{3}\right]$ is the mean queue length. And $E\left[C_{3}\right]$ is the existing queue length until the end of the threshold value of the low priority packet.

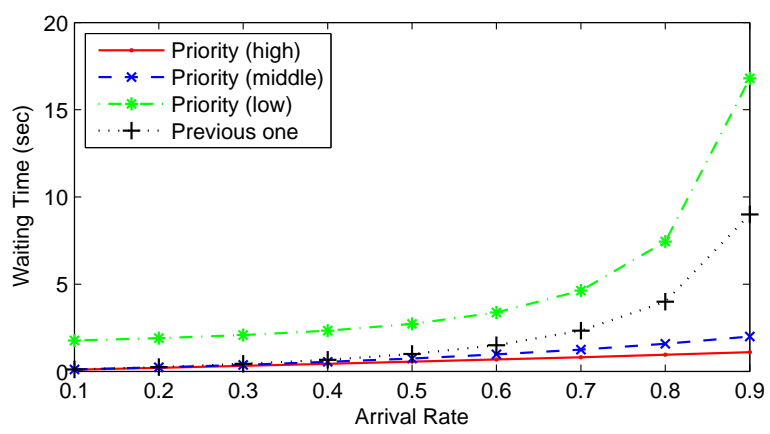

Fig. 5. Waiting time of the proposed method and the previous method

$$
E\left[W_{t}\right]=\frac{E\left[C_{3}\right]}{\lambda_{3}}
$$

where $E\left[W_{t}\right]$ is the delay time that the low priority packet satisfies until the threshold value.

$$
E\left[C_{3}^{\prime}\right]=\lambda_{3} T_{s}-E\left[m_{3}\right]
$$

where $T_{s}$ is the selecting timer value and $E\left[C_{3}^{\prime}\right]$ is the existing queue length until the timer is expired.

$$
E\left[W_{t}^{\prime}\right]=\frac{E\left[C_{3}^{\prime}\right]}{\lambda_{3}}
$$

where $E\left[W_{t}^{\prime}\right]$ is the delay time until the timer is expired.

$$
E\left[A_{2}\right]=\min \left\{E\left[W_{t}\right], E\left[W_{t}^{\prime}\right]\right\} .
$$

According to equation (9), we can find the additional mean delay time in the low priority buffer. Also, we should find the incoming service time of the high and the middle priority traffic during additional delay time. It is according to equation (10).

$$
\sum_{k=1}^{2} E\left[T_{k}^{\prime \prime}\right]=\sum_{k=1}^{2} \frac{\lambda_{k}}{\mu} E\left[A_{3}\right]=\sum_{k=1}^{2} \rho_{k} E\left[A_{2}\right]
$$

Finally, we obtained the graph of the waiting time by calculating each priority packet through the equation (1) to (10).

Fig. 5 shows that the proposed one has the different waiting time according to each service type and the previous method has the same waiting time of all type of services. Arrival rate $\lambda$ of the proposed method and previous method selected each from 0.1 to 0.9 and service rate $\rho$ is considered as $1 . \lambda_{1}, \lambda_{2}$, and $\lambda_{3}$ were indicated the arrival rate of the high, the middle, and the low priority packet, respectively.

As a result, the waiting time of the low priority in the proposed method is longer than the previous method. However, the low priority packet which indicated the non-real time service is tolerant to delay or jitter. Therefore, it does not considered as QoS degradation. On the contrary, we can see that the waiting time of the high and the middle priority packet 
which indicated the real time packet is much shorter than the previous waiting time. That is, the proposed method achieves a significant QoS benefit due to the reduced waiting time waiting time for the real time service.

\section{CONClusion}

The rapid increase in Internet data services over the past decade has led to increased traffics which consist of a real time service and a non-real time service. Particularly, the real time service is more sensitive to QoS factors such as delay and jitter than the non-real time service. In this paper, we propose a QoS method for the real time service by using prioritybased packet classification in HG. Generally, The real time service is sensitive to QoS factors such as delay and jitter. On the contrary, The non-real time service is tolerant to above mentioned QoS factors. To improve QoS for the real time service, UDP is granted a high priority compared with TCP in a data buffer part of HG. We analyze the expected waiting time of priority-based packet by using queueing analysis. The result shows that a significant QoS benefit due to the reduced waiting time for the real time service.

\section{ACKNOWLEDGMENT}

This work was supported by the IT R\&D program of MSIP/KEIT. [10039160, Research on Core Technologies for Self-Management of Energy Consumption in Wired and Wireless Networks]

†Corresponding author is S.G. Choi (sgchoi@cbnu.ac.kr).

\section{REFERENCES}

[1] H. Yang, M. J. Yu, D. G. Yun, and S. G. Choi, "Energy saving mechanism using sleep mode of processor in home gateway," in Consumer Electronics (ICCE), 2012 IEEE International Conference on. IEEE, 2012, pp. 704-705.

[2] S. Y. Ban, J. K. Choi, and H.-S. Kim, "Efficient end-to-end qos mechanism using egress node resource prediction in ngn network," in Advanced Communication Technology, 2006. ICACT 2006. The 8th International Conference, vol. 1. IEEE, 2006, pp. 480-486.

[3] Y. Liu, D. Xu, Z. Mu, and J. Qin, "Efficient hybrid packet classification in traffic control system using network processors," in Advanced Computer Control, 2009. ICACC'09. International Conference on. IEEE, 2009, pp. $57-61$

[4] S. H. Shaikot and M. S. Kim, "Lightweight traffic-aware packet classification for continuous operation," in Applications and the Internet (SAINT), 2010 10th IEEE/IPSJ International Symposium on. IEEE, 2010, pp. 59-67.

[5] K. In-Yeup, "TASA: Traffic aware sleep algorithm for energy-efficient always-on home networks," IJCSNS, vol. 10, no. 6, p. 105, 2010.

[6] M. W. Kim, W. S. Choi, D. G. Yun, J. M. Lee, and S. G. Choi, "A method on reducing processing time using signature matching in a router," in Advanced Communication Technology (ICACT), 2012 14th International Conference on. IEEE, 2012, pp. 726-729.

[7] M. Schwartz, Telecommunication networks: protocols, modeling and analysis. Addison-Wesley Reading, 1987, vol. 7.

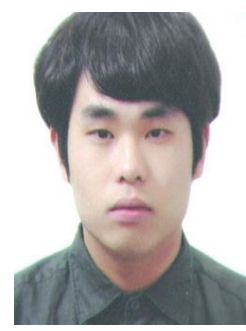

Kyeong Chae Jeong received B.S degree in School of Electrical and Computer Engineering, Chnungbuk National University, Korea in 2013. He is currently a M.S. candidate in School of Electrical and Computer Engineering, Chnungbuk National University. His research interests include NGN, green network, and LTE system.

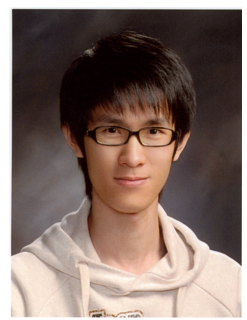

Ju No Han received B.S. degree in College of Electrical \& Computer Engineering, Chungbuk National University, Korea in 2012. He is currently a M.S candidate in College of Electrical \& Computer Engineering, Chungbuk National University. His research interests include green network, mobile management, and LTE system.

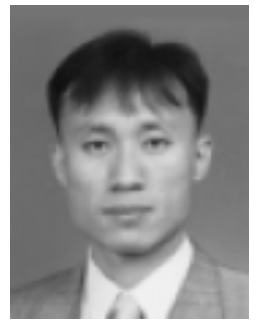

Seong Gon Choi received B.S. degree in Electronics Engineering from Kyeongbuk National University in 1990, and M.S. and Ph.D. degree from Korea Advanced Institute of Science and Technology (KAIST) in 1999 and 2004, respectively. Currently, he is an associate professor in College of Electrical \& Computer Engineering, Chungbuk National University. His research interests include mobile communication, green networks, smart grid (future power grid), high-speed network architectures and protocols. 